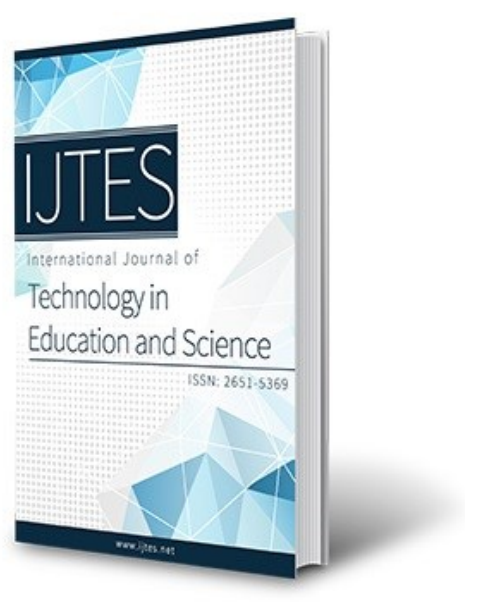

\title{
Enhancing Social Justice: A Virtual Reality and Artificial Intelligence Model
}

Ehi E. Aimiuwu

Campbellsville University, United States of America

www.ijtes.net

\section{To cite this article:}

Aimiuwu, E. E. (2022). Enhancing social justice: A virtual reality and artificial intelligence model. International Journal of Technology in Education and Science (IJTES), 6(1), 32-43. https://doi.org/10.46328/ijtes.331

The International Journal of Technology in Education and Science (IJTES) is a peer-reviewed scholarly online journal. This article may be used for research, teaching, and private study purposes. Authors alone are responsible for the contents of their articles. The journal owns the copyright of the articles. The publisher shall not be liable for any loss, actions, claims, proceedings, demand, or costs or damages whatsoever or howsoever caused arising directly or indirectly in connection with or arising out of the use of the research material. All authors are requested to disclose any actual or potential conflict of interest including any financial, personal or other relationships with other people or organizations regarding the submitted work. 


\title{
Enhancing Social Justice: A Virtual Reality and Artificial Intelligence Model
}

\author{
Ehi E. Aimiuwu
}

\begin{tabular}{ll}
\hline Article Info & Abstract \\
\cline { 3 - 3 } $\begin{array}{l}\text { Article History } \\
\text { Received: }\end{array}$ & CDC.gov (2020) shows that the coronavirus (COVID-19) has exposed the cost of \\
13 August 2021 & an unjust law enforcement and judicial system against minorities. Non-Asian \\
Accepted: & minorities, who are usually the poor with health issues, have been the most \\
25 December 2021 & negatively affected by COVID-19. The aim of this study is to explain through a \\
& literature review how virtual reality (VR) and artificial intelligence (AI) can be \\
& used to enhance social justice in order to eradicate the biases that deny targeted \\
& people access to resources or freedom through the judicial system. Research \\
Keywords & shows that VR is excellent for capturing multi-dimensional and multisensory \\
Artificial intelligence & videos, sounds, and pictures that can help police and judges to conclude exactly \\
Ludicial system & what happened. Also, AI can help to match an incident to past cases or use rules \\
Social justice & of expert systems to predict judgement for new cases, in order for every person \\
Virtual reality & to receive the same judgement and sentencing as a white person involved in a \\
& similar incident.
\end{tabular}

\section{Introduction}

In 2020, there was a worldwide protest against police brutality because of brutality and the killings of unarmed minorities of color at the hands of law enforcement, and unjust incarceration of minorities of color by the judicial system of the United States. NAACP.org (2020) states that incarcerated people have about a 50\% probability of being unemployed and are five times more likely to get infectious diseases compared to others. Furthermore, spending for prisons has increased to about three times the spending for grade schools. This police brutality and these incarcerations not only lead to loss of freedom and voting rights to elect political officers or leaders that can represent their communities and livelihoods effectively, but also to loss of employment and stable family homes, which ultimately leads to poverty. Research from Fathers.com (2014) shows that about $63 \%-90 \%$ of children from fatherless homes are affected by poverty, crime, illiteracy, unemployment, suicide, teenage pregnancy, drugs, sexually transmitted diseases, low life-expectancy, delinquency, or are high school drop-outs. According to Stewart (2019), about 71\% of black women were unmarried, and NAACP.org shows that one in six black males will be missing or dead due to poor healthcare and poverty created by incarceration. Continuous and systemic injustice from law enforcement and the judicial system is a plague in every society and should be eradicated, with immediate effect.

Poverty, which is lack of access to resources or lack of freedom to easily enhance one's socioeconomic status, in 
America stems from the racial profiling of minorities of color by law enforcement and biased judicial systems, which are based on old Jim Crow laws (Black Codes) against blacks and strict immigration policies against Hispanics (NAACP.org, 2020). Nellis (2016) states that whites make up about $62 \%$ of the American population, but about $35 \%$ of the prison population, while Hispanics make up $17 \%$ and blacks make up about $13 \%$ of the population (total of $30 \%$ ), but Hispanics are $21 \%$ and blacks are $38 \%$ of the prison population (total of $59 \%$ ). Black males are incarcerated 5.1 times more than white males, Hispanic males 1.4 times than white males, and black females 2.0 times more than white females (NAACP.org, 2020; Nellis, 2016). King (2017) states that black males serve $19.1 \%$ longer prison sentences than white males. Minorities of color make up about $30 \%$ of the American population, but about $60 \%$ of the prison population, which is the reverse of the white population's reality, and are given about $20 \%$ longer prison sentences by judges. This is a problem that must be alleviated or eradicated, with the help of modern technology, because advancement in modern technology is meaningless if all peoples cannot be free to enjoy all the possibilities from technology through equal access to resources.

Since the incarcerated are most likely to get infectious diseases due to lack of adequate resources, employment, education, and health insurance, we need to find ways to achieve social justice in law enforcement and the judicial system in order to alleviate these social ills suffered by the targeted minorities of color, and increase their ethnic capital and access to resources. CDC.gov (2020) shows that minorities of color suffered most from COVID-19 in the United States in both deaths as well as infections, being infected by COVID-19 1.4-1.8 more times than whites, whereas Asians were 0.6 more times; were hospitalized 3.7-4.1 more times than whites, whereas Asians were 1.2 more times; and died from the disease 2.6-2.8 more times than whites, whereas Asians died 1.1 more times, due to socioeconomic factors, types of occupation, and access to healthcare. This is because non-Asian minorities are the targeted group for incarceration, which leads to high unemployment, illiteracy, poverty, and inadequate healthcare. Figure 1, 2, and 3 show the negative effect of social injustice on the incomes, employment, and health of non-Asian minorities respectively in the United States.

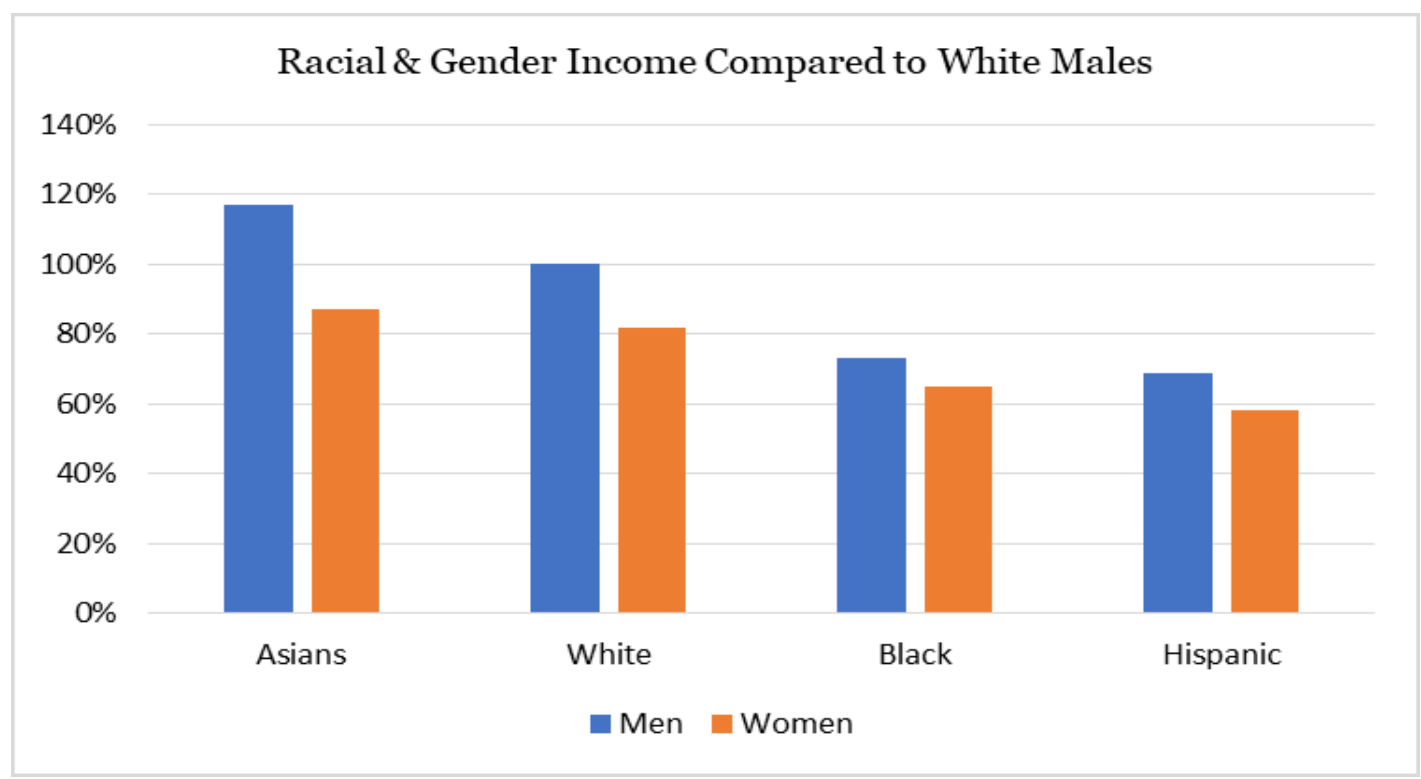

Figure 1. Income Gaps Based on Race \& Gender in the United States (Patten, 2016) 
Guo (2016) states that both whites and Asians are not affected by the old Jim Crow laws (Black Codes) or immigration laws that affect non-Asian minorities, and not only did Asian-Americans not surpass other minorities until after World War II as the model minority that never complained, but white Americans stopped being racist towards Asians in order to win the Cold War and be leader of the free world. Today, and over 50 years after World War II, Asians are now even more productive socioeconomically than whites. Figures 1 and 2 also show that eliminating systemic discrimination or bias from law enforcement and the judicial system would allow all minorities and women to enjoy the freedom or equal access to resources, which both whites and Asians enjoy in America today. Guo (2016) further states that Asian Americans were the least well-off of the minorities in the United States over 50 years ago, but now have the highest income and lowest unemployment rates among all races and genders in America. The two figures also show that those without restrictive laws tend to prosper better than those that enforce restrictive laws and those affected by the restrictive laws. We do not have to convince the European and Asian nations only that capitalism is better than communism, but, rather, convince the entire world that all people can achieve optimum productivity when they have equal access to resources, freedom, and power.

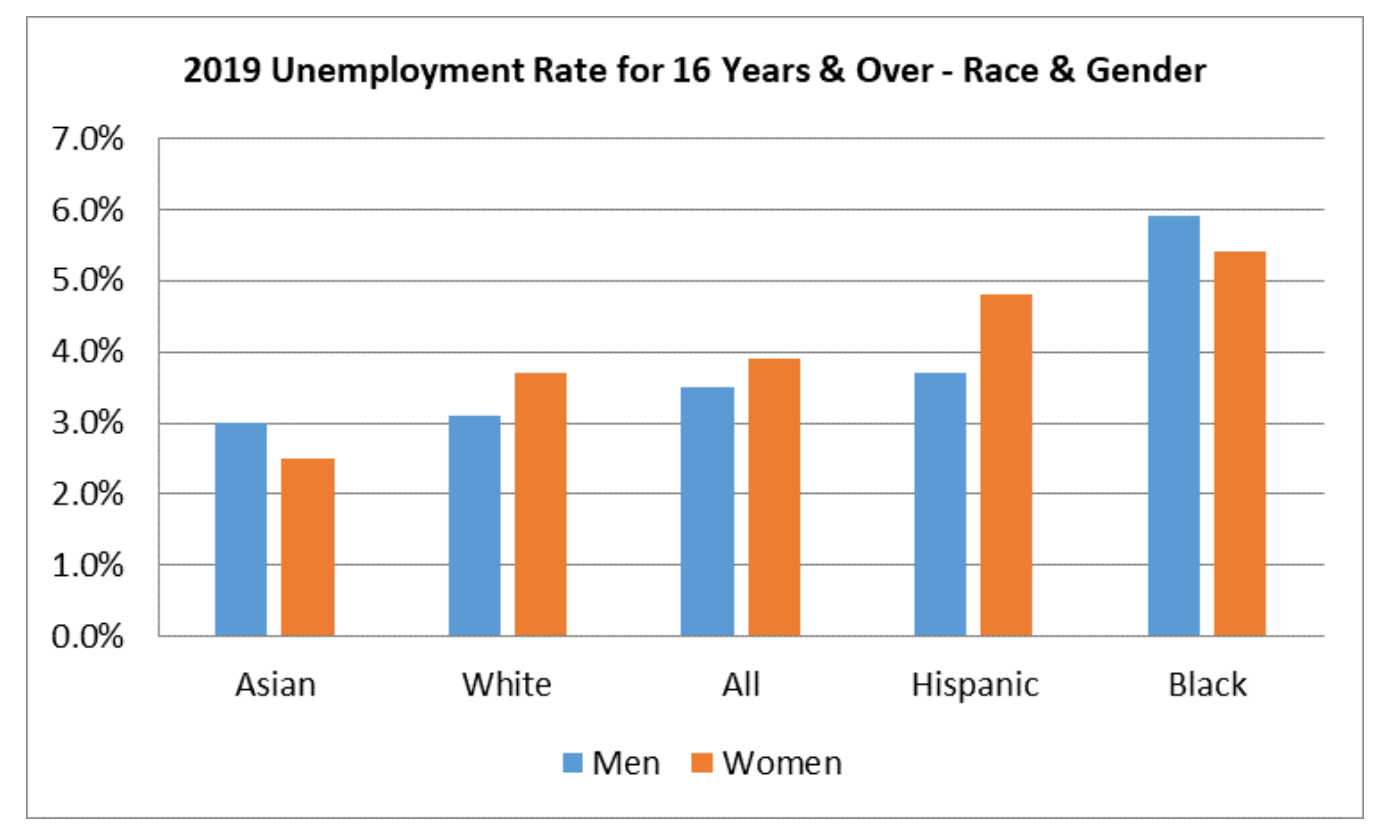

Figure 2. United States Unemployment Rate for 2019 (BLS.gov, 2019)

The menace of COVID-19 in the United States revealed that racism is fueled by the failure of both law enforcement and the judicial system to provide adequate justice, which has led to the poverty that ultimately leads to poor healthcare and death. Groups that have low ethnic capital or are legally denied access to power and adequate healthcare will be discriminated against and undermined by others. Despite the fact that research has been done on both VR and AI for both law enforcement and the judicial system, very little research has been done to combine both VR and AI as a strategy to provide social justice and equal access to resources for all people. The rest of this paper will discuss both VR and AI, as well as present VR and AI models that can help to enhance social justice in both law enforcement and the judicial system. The models will be merged together as well as discussed, and limitations will be presented, followed by the conclusion. 


\section{Literature Review}

\section{Virtual Reality}

VR is the combination of both hardware and software systems that possess a unique ability to make users have telepresence while immersed and interacting in another environment (Mutterlein, 2018). The goal of VR is to use the technology of integrated devices through users' five senses to give them a multisensory experience of feeling close to a different and realistic reality, which also allows users to change their perception of the world, as well as provide the capacity to store, transmit, or share information in a timely fashion (Pinto et al., 2019; Carlson \& Caporuso, 2018). VR needs to be multi-dimensional with adequate sounds and colors that match the physical environment in order to give all human senses a real and authentic feeling.

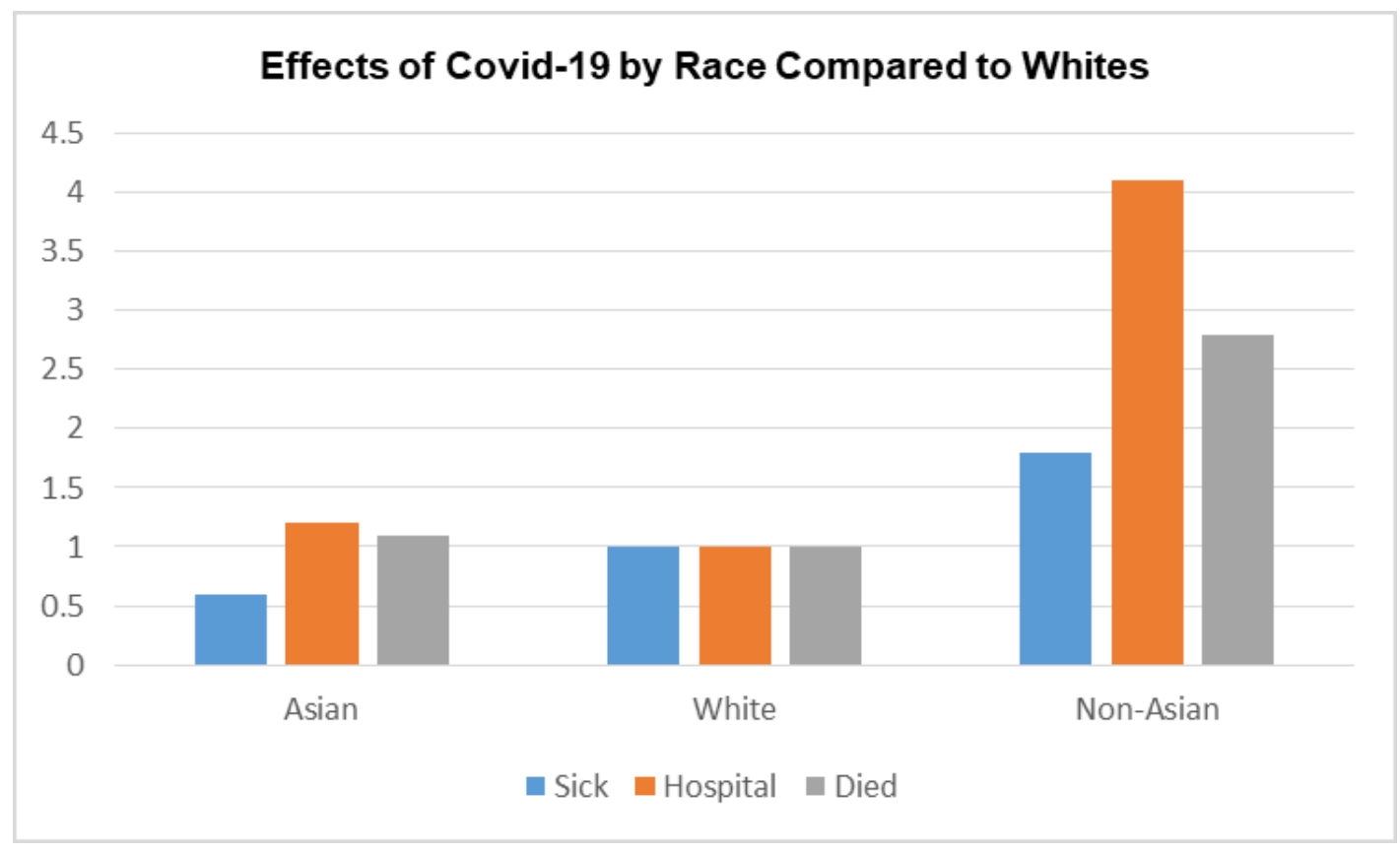

Figure 3. United States Covid-19 Rate by Race (CDC.gov, 2020)

Three dimensional (3D) GIS is a form of VR that provides visualization, interaction, navigation, and accurate analysis of urban or underground infrastructure to support timely decision making on both geospatial and spatial data for direct integration of 3D images into web pages in the fields of transportation, telecoms, the urban environment, and agriculture (Jurado et al., 2017). 3D GIS tends to be more realistic because it has in-depth representation and enhanced visualization, which leads to the creation of 3D scenarios and overlapping spatial datasets, such as measurements beside trees and buildings (Jurado et al., 2017; Carlson \& Caporuso, 2018). If these 3D technologies can help the user to perceive what may be going on underground and beside trees as well as buildings, they may be very helpful to law enforcement in investigating incidents when there is lack of witnesses or both cameras and microphones were insufficient. They can also help law enforcement to predict or conclude objectively what may have occurred in real time without any bias.

VR is used by many United States agencies, including the Department of Homeland Security, the Center for Disease Control and Prevention, and police departments to support emergency responses and multi-agency 
collaboration, due to its advancements in motion capture technology and zero-latency cameras that allow physically immersed virtual reality (Carlson \& Caporuso, 2018). This shows that VR has been accepted as reliable in national defense, investigation, health, and emergency response fields, but it does not show that VR is being used for social justice in combination with AI.

The three key features possessed by VR are telepresence, immersion, and interactivity, where presence is a subjective experience of being at a particular location when you are physically located in another, telepresence is a state of presence while using a medium, immersion is a psychological state of mind or optimal experience where the user is completely absorbed in an activity within the medium, interactivity is the psychological state of mind or the degree by which a user influences or manipulates the content of the medium, and satisfaction is the user's feeling about an actual experience with a product or service (Mutterlein, 2018). Interactivity strongly influences both telepresence and immersion, telepresence strongly influences immersion, and immersion strongly influences satisfaction, as shown in Figure 4 (Mutterlein, 2018). Study shows that users felt more presence (telepresence) and satisfied when they used VR for learning than just audio because they felt more involvement and spatial presence, as well as experienced realism (Pinto et al., 2019). For VR to be valuable to the user, the user must be able to influence or manipulate the $3 \mathrm{D}$ content to get their desired result through telepresence, which keeps them immersed within the medium to generate some form of satisfaction.

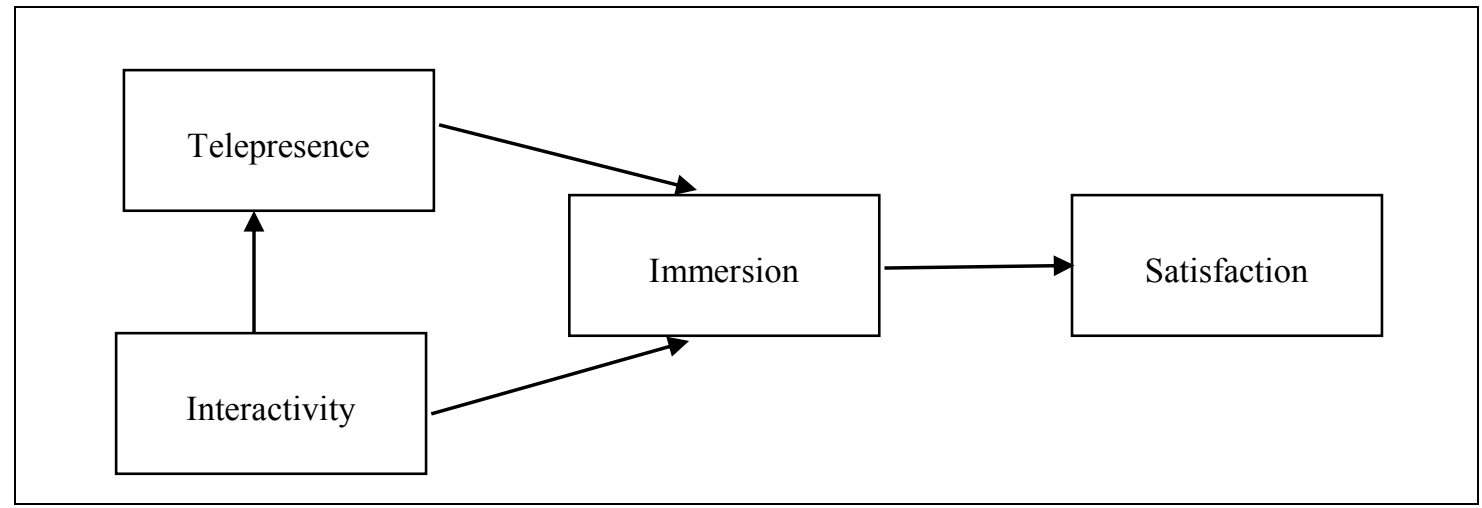

Figure 4. Three Key Features of Virtual Reality (Mutterlein, 2018)

\section{Artificial Intelligence}

$\mathrm{AI}$ is an intelligent system of tools, techniques, and algorithms that has the ability to think and learn as well as augment work, which includes natural language processing (analyzing human language), machine learning (algorithms for learning), and machine vision (algorithms for image analysis) (Jarrahi, 2018). AI can learn from past experiences and data to develop intelligent solutions, can learn to improve itself for knowledge-based tasks, and is useful for analytical decision making, while humans are useful for intuitive decision making (Jarrahi, 2018). AI can get VR 3D data to analyze the image, sound, colors, and activities as well as recreate the probable incident that may had occurred objectively from learning the time, distance, weight, and speed of the objects involved for easier decision making by law enforcement at the scene of an incident, and judges in the courtrooms, without bias. 
The analytical approach of AI requires analysis of knowledge based on conscious reasoning and logical deliberation, but lacks understanding of common-sense and unpredictable situations, while the intuitive approach of humans is based on business instinct, past experiences, and gut feeling, but has the advantage of creativity and imagination in decision making (Jarrahi, 2018). It is best to merge the ability of AI to analyze vast amounts of data in real time with superior human intuition and insight for judgement, which is also called hybrid intelligence (HI), as shown in Figure 5 (Jarrahi, 2018; Dellermann et al., 2019). HI will be necessary to reach a satisfactory and unbiased decision for all members involved when the facts of an incident are going to be based on the recreated or predictive incident provided by AI from VR.

AI helps to improve human decisions by providing predictions, while humans can help AI to learn through updated machine learning models, so HI allows humans to benefit from the predictive ability of AI, and humans then use their intuition, creativity, and imagination to make decisions based on AI's predictions that are without bias, such as racism or sexism (Dellermann et al., 2019). While AI will provide a prediction close to an actual incident that occurred in real time, it will be the law enforcement officer and judge (humans) that set the learning model that the AI's predictive ability will be based upon, and the officer and judge will also make the final decision based on the recommendations from past behaviors or rulings from previous human experts. An expert system is a part of AI or a type of computerized HI that requires the emulation of human expert behavior by using human experts as both data and production rules in a computer program which can be used to solve very complex issues (Abu-Nasser, 2017; Campbell, 2020). Based on previous experts' decisions or rulings provided by AI, an officer at the scene or judge in the courtroom can use AI's recreated prediction of an actual incident to match the exact decision made by a previous expert about the exact same situation without bias, and the AI, through HI, learns to update its predictive ability, based on the data from VR and HI for quicker and easier decision making in the future.

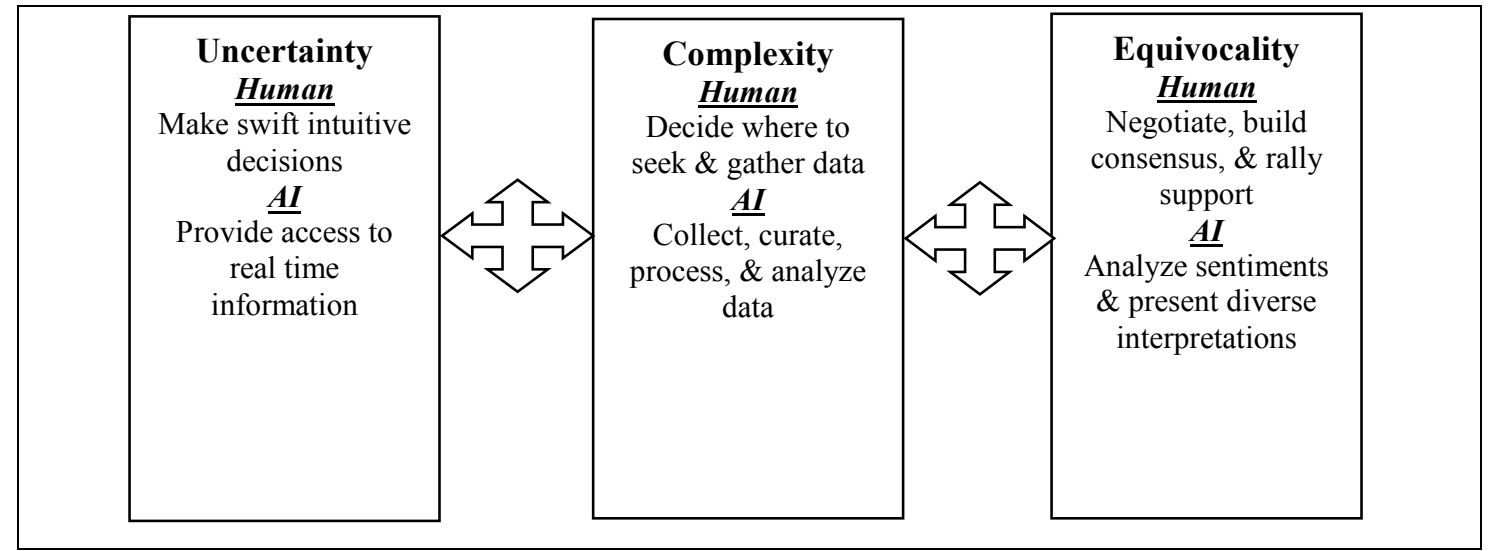

Figure 5. Hybrid Intelligence (Human \& AI) Decision Making Situations (Jarrahi, 2018)

AI is being used regularly around the world by lawyers, police, and judges because it has tools for visualizing arguments (Araucaria, DART, and QuestMap), for case-based reasoning (Judge and ASSYST) and abductive reasoning (ECHO and ALIAS) in an attempt to provide objective judgements (Nissan, 2017). Case-based or data-reliant reasoning uses past cases to determine solutions for a new case through relationships, patterns, and correlations, or explain how it does not fit, whereas the judicial system uses models of judges' decisions when 
sentencing, and ASSYST is an expert system aimed at enhancing uniform sentencing. Abduction reasoning or rules-based reasoning is a form of theory or interpretative inference that first describes the data to explain the data before sentencing with the help of expert systems, which makes it logic based (Nissan, 2017; Campbell, 2020). Biometrics is identifying individuals based on their physiological or behavioral qualities, which can verify an identity or authenticate the identity in question (Nissan, 2017). AI is used to accelerate legal research for both lawyers and judges, as well as whether prisoners should be released at pretrial, to determine the level of bail amount, and to ensure that all courts have similar resolutions for similar facts and similar legal conditions (Campbell, 2020). AI is a great tool for social justice for all peoples by ensuring that everyone gets the same sentence or judgement as a white person or rich and powerful individual, in a similar situation, in all places.

\section{Methodology}

Since technology has being useful in exposing police brutality in real time through smartphone cameras, as well as on social media, and civil rights activists have been consistent in demanding a change to the unjust judicial system through the news outlets, it was best to include research and data from governmental and civil rights organizations in this paper. A total of 18 sources were used to show why social justice is needed desperately at this time of daily killings of minorities of color. Two civil rights articles were used to provide data on both police brutality and uneven sentencing by the courts, four news sources were explored to show the negative effects of injustice on the lives of minorities of color, and two governmental sources were used to study how injustice affects both the labor and health of minorities of color. Six articles were used to understand what AI is, how it is used by police and the courts, and how it can possibly be used to combat injustice from both the police and courts. Four articles were used to understand what VR is, its benefits, and how it can also be used to combat social injustice. This paper is not just trying to present evidence of social injustice against minorities of color, but is also attempting to show how injustices negatively affect the labor, ethnic capital, as well as health of minorities of color, and how technology can be used to combat social injustice from both the police and the courts through VR and AI.

\section{Relationship and Model}

The issue of privacy in VR may be of concern to individuals and businesses in the investigated area, but we already see in the media, police bodycams displaying exactly what happens at crime scenes. Also, the issue of bias in historical AI data may be a concern in the use of case-based reasoning that led to an unfavorable and unjust decision in the past being used again. Biased law enforcers and judges may want to use AI rule-based models that intentionally rules against minorities of color.

To eliminate these concerns, especially at a time of almost daily police brutality and injustices against minorities of color, it may be best that we establish an integrated data sharing protocol to encourage digital device users (individuals and businesses) to allow their digital data (videos, images, and sounds) be used by media, law enforcement, and courts when needed for an investigation at only the specific time and location of the investigated coordinates, with privacy controls. This means that only data for a specific time and location can be 
pulled into the investigator's VR for analysis and should only be used to investigate the incident in question. As for biases in AI, only case-based and rule-based reasoning models for whites in a similar situation from the past will be used, in order to get an equal and fair decision for all people.

The model in Figure 6 is based on the combined models in Figure 4 (Features of VR) and Figure 5 (HI decision making), which is to combine VR and AI to achieve social justice for everybody, everywhere. There are a total of seven stages. Stages 1-3 are for VR, stages 4-6 are for AI, and stage 7 is for the satisfaction from the first six stages. The first three stages show how law enforcement and the judicial system may use VR. The second three stages show how AI may be used in conjunction with VR. Stage 7 shows how satisfied law enforcement officers and judges may be, as well as people in society, with the use of both VR and AI to provide social justice for all, regardless of race, gender, ethnicity, religion, sexual preference, or age. The use of both VR and AI in Figure 6 to enhance social justice is actually a cycle that goes from VR to AI to satisfaction and back to VR. This shows that the support for social justice through the use of both VR and AI together could be support by law enforcement, judges, and people in society, if it leads to a just and fair judgement for all peoples, without any form of bias.

\section{Results}

The combined VR and AI cycle will depend on an integrated data sharing protocol from various networks, systems, and platforms in the future for social justice, as explained in Figure 6 below.

Stage 1 is Interactivity: The user uses VR to retrieve all videos, audio, and pictures from building and street cameras, automobile and cellphone cameras, sounds from digital speakers and microphones (Google Assistant, Amazon's Alexa, or Apple's Siri), as well as pictures about the location of the incident from all angles and directions. This is to aid the user while investigating a complaint or an incident, and corroborating it with what the witnesses, victim, or perpetrator said about the incident.

Stage 2 is Telepresence: The user uses VR to be present at the scene, despite not being there in the physical world or having been there, but needs to verify what was seen and heard to avoid any form of bias. At this level, the multisensory and multi-dimensional qualities of VR are essential. The user is now transplanted to the location of incident while seeing and hearing what occurred as it was occurring. The user is able to emotionally feel the verbal and physical abuse, feel the stab from a knife or punch from the fist, or feel the taste of poisoned drink being consumed or the smell of a pool of blood on the floor.

Stage 3 is Immersion: the VR user is absorbed or focused on the combined effect of both Interactivity and Telepresence, which gives the user the opportunity to match the VR experience with what was investigated or spoken about by witnesses in order to come to an adequate conclusion of what may have taken place at the scene. Also, how, when, where, and what happened is clearly understood.

Stage 4 is Uncertainty: This is the point that the VR user becomes an AI user by combining the VR experience 
with AI in order to arrive at an objective decision without bias. This is where HI begins, because the VR user becomes an AI user that uses human intuition, creativity, and imagination to inform AI about the time, angles, and location of an incident to focus on in order for AI to provide real-time analysis of the incident.

Stage 5 is Complexity: The AI user decides which videos, sounds, and pictures are to be analyzed for decision making, and AI goes ahead to use its analytical abilities to sort through the data to come up with a decision of what the incident was actually all about.

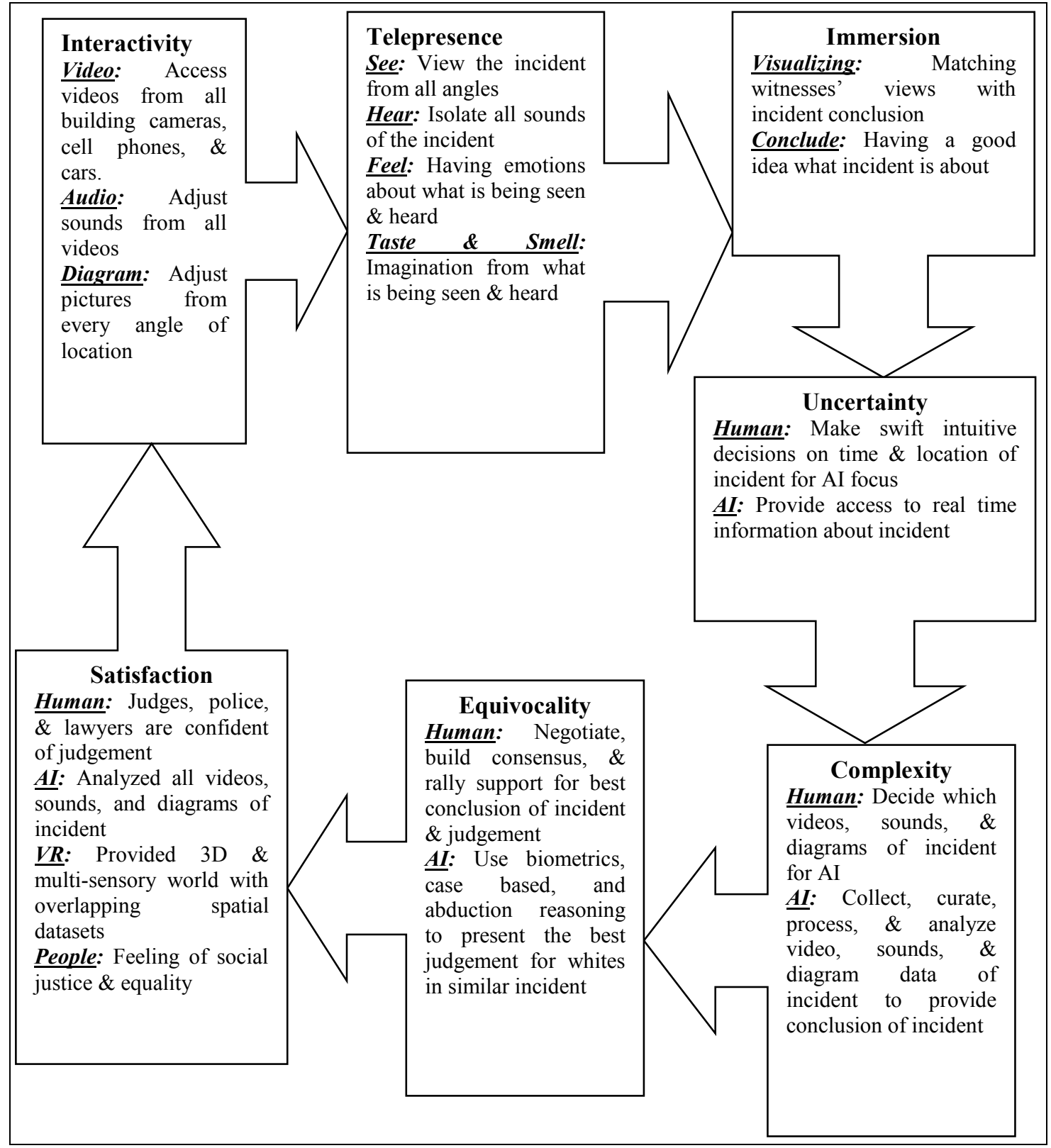

Figure 6. Combining Virtual Reality \& Artificial Intelligence for Social Justice

Stage 6 is Equivocality: AI uses biometrics to decide or authenticate who the abuser or perpetrator is, if it was previously unknown, or to verify the known identity. Then, a case-based or data reliant reasoning is used to find a similar incident in the past and recommend the best judgement that was given a white offender, if a similar 
case was found in that city or state. If a similar case was not found in the past, abductive or rules-based reasoning is used to apply logic or interpretive inferences, based on expert systems that rely on the decision or behavioral pattern of police and judges for white offenders.

Stage 7 is Satisfaction: The VR user is satisfied that the VR has provided all the videos, audios, and pictures required to make an accurate judgement of the incident. The AI user is satisfied and confident that it provided the models for the right location and time of incidents that needed to be analyzed, as well as accurate videos, sounds, and pictures for adequate decision making. The AI user is also satisfied that biometrics are used to verify and authenticate the perpetrator, use case-based reasoning to match the best judgement given to a white offender for a similar incident, or rely on rules-based reasoning to logically make a best judgement for a white offender, based on expert systems of police and judges.

\section{Conclusion}

In summary, the combined use of VR and AI to enhance social justice is a good idea, but must depend on an integrated data sharing protocol that allows integrated data from various networks, platforms, and systems. While many companies and government agencies have been using them separately, or rarely combined, it is best to use both VR and AI to ensure that arrests are justified and just sentences are given, especially to minorities of color in the United States. Unjust and biased arrests and sentencing have not only reduced their ethnic capital, but have also denied these non-Asian minorities access to adequate resources, employment, healthcare, legal representation, education, voting rights, and freedom in general.

The use of both VR and AI ensures that fair and just arrests as well as sentencing are made that is equal to that of whites because whites have a prison population percentage that is about half their national population percentage, while blacks have about three times their national population percentage, and blacks are given about $20 \%$ longer prison sentence than whites. As long as law enforcement officers and judges are trained on how to use the VR and AI systems adequately, are monitored regularly for efficiency, and the expert system models used are based on the best sentencing and judgements for whites with similar incidents and similar facts, both VR and AI will lead to less bias against minorities of color, which currently denies them both resources and freedom. VR and AI should help police to decide on the best arrest actions on the streets, and help lawyers and judges to decide on best judgements for all in the courts.

\section{Limitations}

This study was based solely on a literature review, but it could have been qualitative research where experts of VR and AI were interviewed to explore the legality of using an integrated data sharing protocol between networks, systems, and platforms to acquire the necessary digital data (videos, images, and sounds) from digital street cameras and building cameras, as well as smartphones in the vicinity of investigation. This study assumes an integrated data sharing protocol is possible in the near future, especially at this time of extreme daily brutality against colored minorities by American police, and the fact that police already use authorized or volunteered 
data from buildings, cars, and people.

A limitation is the bias of the human users of both VR and AI, as well as of the humans who created the AI models on which AI will make its predictive recommendations, and not the VR or AI system itself. If the biased officer or judge using the VR intentionally chooses the wrong angle or location coordinates, a user can claim insufficient evidence and dismiss the case. A biased AI user can also do the same to claim that an innocent passerby may be the perpetrator of the crime or use a poor quality audio to say there is no evidence of a scream or gunshot, so verification by witnesses and accused at the scene is essential for both VR and AI satisfaction.

With AI, a user may choose to use rules-based reasoning instead of case-based reasoning to make it appear that it is new kind of case, and use system experts based on judges that are racist or sexist. Case-based reasoning for white offenders is best for fairness and equality. VR and AI data should be public and available to all, especially the accused, for authenticity and credibility of both VR and AI.

Some may argue that using a judgement based on only white offenders may be racist, sexist, and subverting the quality of the law, but the purpose of both VR and $\mathrm{AI}$ in this study is to ensure that all get the best and equal treatment under the law as expected. This means that both law enforcement officers and judges either have to start allowing equal justice for all or we start terminating those who are racist and sexist immediately by law. This would allow all people to move out of the shackles of restrictive laws in order to have equal access to resources and freedom, as well as increase their ethnic capital, just like the Asians have done in the United States.

\section{References}

Abu-Nasser, B. (2017). Medical expert systems survey. International Journal of Engineering and Information Systems (IJEAIS), 1(7), 218-224.

Bureau of Labor Statistics (2019). Labor force statistics from the current population. Retrieved from http://bls.gov

Campbell, R. W. (2020). Artificial intelligence in the courtroom: The delivery of justice in the age of machine learning. Colorado Technology Law Journal, (18), 323.

Carlson, G., \& Caporusso, N. (2018). A physically immersive platform for training emergency responders and law enforcement officers. In: International Conference on Applied Human Factors and Ergonomics, Springer, Cham, pp. 108-116.

Center for Disease Control (2020). COVID-19 hospitalization and deaths by race and ethnicity. Retrieved from http://cdc.gov/

Dellermann D., Ebel P., Söllner M., \& Leimeister, J. M. (2019). Hybrid intelligence. Business \& Information Systems Engineering, 61(5), 637-643.

Guo, J. (2016). The real reasons the U.S. became less racist toward Asian Americans. Retrieved from http://washingtonpost.com 
Jarrahi, M. H. (2018). Artificial intelligence and the future of work: Human-AI symbiosis in organizational decision making. Business Horizons, 61(4), 577-586.

Jurado, J. M., Graciano, A., Ortega, L., \& Feito, F.R. (2017). Web-based GIS application for real-time interaction of underground infrastructure through virtual reality. In: Proceedings of the 25th ACM SIGSPATIAL International Conference on Advances in Geographic Information Systems, pp. 1-4.

King, E. Y. (2017). Black men get longer prison sentences than white men for the same crime: Study. Retrieved from http://ABCnews.go.com

Mütterlein, J. (2018). The three pillars of virtual reality? Investigating the roles of immersion, presence, and Interactivity. In: Proceedings of the 51st Hawaii International Conference on System Sciences.

National Association for the Advancement of Colored People (2019). Criminal justice fact check. Retrieved from http://naacp.org

National Center for Fathering (2014). The extent of fatherlessness. Retrieved from http://fathers.com

Nellis, A. (2016). The color of justice: Racial and ethnic disparity in state prisons. http://sentencingproject.org

Nissan, E. (2017). Digital technologies and artificial intelligence's present and foreseeable impact on lawyering, judging, policing and law enforcement. AI \& Society, 32(3), 441-464.

Patten, E. (2016). Racial, gender wage gaps persist in U.S. despite some progress. Retrieved from http://pewresearch.org

Pinto, D., Peixoto, B., Krassmann, A., Melo, M., Cabral, L., \& Bessa, M. (2019). Virtual reality in education: Learning a foreign language. In: World Conference on Information Systems and Technologies, Springer, Cham, pp. 589-597.

Stewart, D. M. (2019). 2019 marked 400 years of 'forbidden black love' in America. Retrieved from http://washingtonpost.com

\section{Author Information}

\section{Ehi E. Aimiuwu, Ph.D.}

(iD) https://orcid.org/0000-0002-6272-5782

Campbellsville University

1 University Drive, Campbellsville, KY. 42718

United States of America

Contact e-mail:eeaimiuwu@campbellsville.edu 